\title{
Producción ISI y tramos de investigación: cómo combinarlos en un nuevo indicador
}

\author{
Por Grupo Scimago
}

Grupo Scimago. «Producción ISI y tramos de investigación: cómo combinarlos en un nuevo indicador". En: El profesional de la información, 2006, mayo-junio, v. 15, n. 3, pp. 227-228.

COMO EN LA MAYOR PARTE DE LOS PAÍSES INDUSTRIALIZADOS, en España existe una agencia cuyo principal objetivo es evaluar y otorgar incentivos a la investigación científica: la Comisión Nacional de Evaluación de la Actividad Investigadora (Cneai) ${ }^{1}$.

Fue creada en 1989, dependiente del Ministerio de Educación y Ciencia, y entre sus actividades figura la evaluación y otorgamiento de incentivos económicos para la producción científica de los profesores universitarios. Llamados coloquialmente tramos, se otorgan por períodos de seis años, por lo que también son conocidos como sexenios. Para un estudio más detallado de la Cneai y su efecto en la ciencia española de los últimos años, se recomienda el estudio de Jiménez, Moya y Delgado ${ }^{2}$.

Si bien los tramos de investigación se otorgan a los investigadores a título personal, podemos contar también los que acumula la institución, por lo que este dato se convierte así en un indicador del nivel de excelencia institucional. A pesar de ello no es muy usado en los estudios bibliométricos tradicionales, ya que se encuentra más extendida la utilización de la producción en las bases de datos del Institute for Scientific Information (ISI) ${ }^{3}$. Últimamente algunos informes de la ciencia recogen tanto los primeros como los segundos ${ }^{4}$.

Ahora bien, con estos dos indicadores en la mano la pregunta obligada que nos debemos hacer es si existe alguna relación entre los tramos de investigación que recibe una universidad y su producción ISI. Para ello, en este trabajo compararemos los indicadores de producción ISI de las universidades españolas, con la cantidad de tramos de investigación que la Cneai ha otorgado a sus profesores.

Los datos han sido extraídos del Atlas de la Ciencia Española y consisten básicamente en la producción ISI y los tramos de todas las universidades públicas españolas.

Se representaron en una gráfica de dispersión con el fin de determinar su grado de correlación. En la figura 1 podemos observar que efectivamente existe una alta correlación entre ambos indicadores con un $\mathrm{R}^{2}=0,9014$. Esto se debe en primer lugar a que son sensibles al tamaño de las instituciones y además a que la Cneai tiende a premiar la producción ISI de un investigador mediante tramos.

Sin embargo existen universidades que se apartan claramente de la línea de tendencia. Aquellas que se encuentran sobre ella presentan una producción ISI infravalorada en términos de tramos de investigación (claramente la $U B$ ). Por otra parte, las que se encuentran debajo, presentan una producción ISI sobrevalorada en relación a sus tramos (p. ej. la UCM).

Esto no quiere decir que la Cneai trate mal a aquellas universidades que se encuentran por encima, ni que sea benevolente con las de debajo. La explicación de este fenómeno se encuentra quizás en la composición temática de la producción de cada institución, ya que un tramo se otorga por cinco publicaciones (normalmente $I S I$ ), indepen-

\begin{tabular}{|c|c|c|}
\hline Siglas & Nombre & P/T \\
\hline$U B$ & Universitat de Barcelona & 5,797783354 \\
\hline$U A B$ & $\begin{array}{l}\text { Universitat Autónoma de } \\
\text { Barcelona }\end{array}$ & 5,301470525 \\
\hline UPV & $\begin{array}{l}\text { Universidad Politécnica } \\
\text { de Valencia }\end{array}$ & 4,850736707 \\
\hline USC & $\begin{array}{l}\text { Universidad de Santiago } \\
\text { de Compostela }\end{array}$ & 4,775379696 \\
\hline$U A$ & Alicante & 4,641333749 \\
\hline Uvigo & Vigo & 4,592467083 \\
\hline UJI & $\begin{array}{l}\text { Universitat Jaume I } \\
\text { Castellón }\end{array}$ & 4,549336487 \\
\hline URV & Universitat Rovira i Virgili & 4,532616618 \\
\hline$U P C$ & Politécnica de Cataluña & 4,521140362 \\
\hline$U A H$ & Alcalá de Henares & 4,514141081 \\
\hline$U A M$ & Autónoma de Madrid & 4,386093533 \\
\hline UPV & País Vasco & 4,231638995 \\
\hline UIB & $\begin{array}{l}\text { Universitat de les Illes } \\
\text { Balears }\end{array}$ & 4,136160098 \\
\hline UNICAN & Cantabria & 4,069909857 \\
\hline UJAEN & Jaen & 4,025362319 \\
\hline UCO & Córdoba & 3,955592507 \\
\hline$U V$ & Universitat de València & 3,94441058 \\
\hline$U A L$ & Almería & 3,91044053 \\
\hline UNIZAR & Zaragoza & 3,89702691 \\
\hline UNIOVI & Oviedo & 3,628665727 \\
\hline$U D G$ & Universitat de Girona & 3,597613571 \\
\hline$U M H$ & $\begin{array}{l}\text { Universidad Miguel } \\
\text { Hernández de Elche }\end{array}$ & 3,591375044 \\
\hline UEX & Extremadura & 3,583826215 \\
\hline Ulpgc & Las Palmas de G. C. & 3,569796374 \\
\hline$U C M$ & Complutense de Madrid & 3,512791142 \\
\hline ULL & La Laguna & 3,39272429 \\
\hline$U M$ & Murcia & 3,386997749 \\
\hline$U D C$ & Universidade da Coruña & 3,354450768 \\
\hline$U G R$ & Granada & 3,289982072 \\
\hline UPM & Politécnica de Madrid & 3,282609346 \\
\hline$U M A$ & Málaga & 3,11207371 \\
\hline Unavarra & $\begin{array}{l}\text { Universidad Pública de } \\
\text { Navarra }\end{array}$ & 3,075965539 \\
\hline$U C A$ & Cádiz & 3,07503075 \\
\hline Unirioja & La Rioja & 3,057987565 \\
\hline$U C L M$ & Castilla-La Mancha & 2,981563996 \\
\hline$U B U$ & Burgos & 2,899470899 \\
\hline UVA & Valladolid & 2,784210526 \\
\hline USAL & Salamanca & 2,764198201 \\
\hline$U H U$ & Huelva & 2,731946437 \\
\hline US & Sevilla & 2,731684156 \\
\hline UPCT & Politécnica de Cartagena & 2,543424318 \\
\hline UPF & $\begin{array}{l}\text { Universitat Pompeu } \\
\text { Fabra }\end{array}$ & 2,43829183 \\
\hline UNILEON & León & 2,292335474 \\
\hline UC3M & Carlos III & 2,077922078 \\
\hline$U D L$ & Universitat de Lleida & 2,038888889 \\
\hline UNED & UNED & 1,576872536 \\
\hline URJC & Rey Juan Carlos & 1,323251418 \\
\hline UPO & Pablo de Olavide & 0,70163005 \\
\hline
\end{tabular}

diente de las que el investigador tenga. De esta forma, habrá áreas temáticas donde los investigadores publicarán sólo cinco trabajos ISI por cada tramo/sexenio, mientras que en otras esta cantidad puede ser muy superior. Incluso existen áreas de conocimiento donde se puede optar a un tramo sin necesidad de 


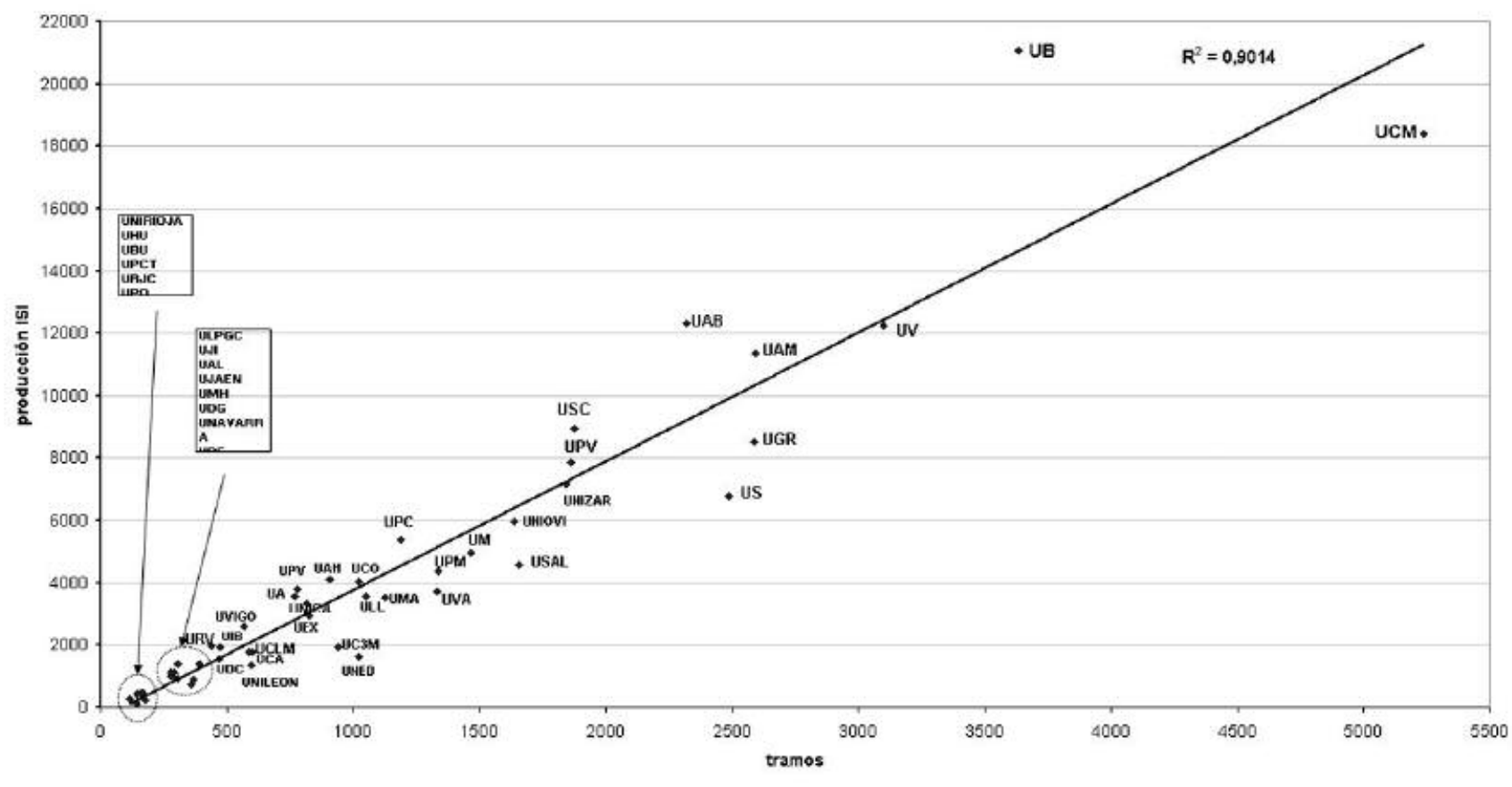

Figura 1

tener publicaciones ISI (humanidades, sociales y jurídicas).

En la tabla vemos un ranking de universidades ordenado por el cociente entre producción ISI y tramos Cneai. A este nuevo indicador lo llamaremos P/T. En las primeras posiciones nos encontramos con las universidades que más se apartan, por arriba, de la línea de tendencia. Incluso se observan con más claridad que en el gráfico, ya que el factor de escala no permite apreciar estas diferencias. El valor medio, por donde pasa la línea de tendencia, se encuentra entre la $U E X$ y la $U M H$ por lo que hemos pintado la tabla con dos tonos diferentes a partir de este punto.

Debemos preguntarnos qué significa realmente este indicador $\mathrm{P} / \mathrm{T}$. $\mathrm{Si}$ tenemos en cuenta que los tramos recibidos por una universidad son un indicador de su excelencia científica y, por otra parte, la producción ISI es un indicador de su visibilidad internacional, $\mathrm{P} / \mathrm{T}$ debe combinar ambas cuestiones; por lo tanto, debe ser, un indicador de la excelencia internacional de una universidad española.

\section{«Si bien los tramos de investigación se otorgan a los investigadores a título personal, podemos contar también los que acumula la institución»}

Esta es una primera y breve aproximación al indicador, aunque creemos que arroja elementos suficientes como para seguir trabajándolo con más detalle. El próximo paso consistirá en estudiarlo para diferentes áreas de conocimiento (ciencias básicas y experimentales vs. ciencias sociales y humanidades), e incluso para las diferentes comisiones Cneai (11 en total).

Otra variable con la que se puede cruzar es el de la tasa de éxito en la concesión de proyecto de investigación ${ }^{6}$. Una tasa de éxito alta combinada con un indicador P/T bajo, podría hacer a una universidad sos- pechosa de ser favorecida por el sistema, y viceversa.

Creemos que de estos estudios con más profundidad se podrá detectar una mayor gama de matices para este nuevo indicador.

\section{Notas}

1. http://wwwn.mec.es/univ/jsp/plantilla.jsp?id=501

2. Jiménez-Contreras, Evaristo; Moya-Anegón, Félix; Delgado López-Cózar, Emilio. The evolution of research activity in Spain. The impact of the National Commission for the Evaluation of Research Activity (Cneai), Research Policy, 32, pp. 123-142, 2003.

3. http://www.isinet.com

4. Moya-Anegón, Félix (dir.); Chinchilla-Rodríguez, Zaida (coord.). Indicadores bibliométricos de la actividad científica española2004. Madrid: Fundación Española para la Ciencia y la Tecnología, 2005 (Observatorio Fecyt de Política Científica y Tecnológica).

5. http://www.atlasofscience.net

6. Eugenia García, Clara; Sanz-Menéndez, Luis. «Competition for funding as an indicator of research competitiveness». En: Scientometrics, 2005, August, v. 64, n. 3, pp. 271-300.

Grupo SCImago (Imago scientiae o visualización de la ciencia).

scimago@ugr.es

http://www.atlasofscience.net 